\title{
Assessment of the Brief ICF Core Set Reliability for the Evaluation of Working Capacity in the Case of Multiple Sclerosis
}

\section{Daiva Valadkevičienė ${ }^{1}$, Dalius Jatužis', Lina Būtènaitè ${ }^{2}$, Remigijus Gulbinas ${ }^{3}$}

${ }^{1}$ Department of Neurology and Neurosurgery, Institute of Clinical Medicine, Vilnius University, Faculty of Medicine, Lithuania

2 Vilnius University hospital Santaros klinikos Rehabilitation, Physical and Sports medicine centre, Lithuania

${ }^{3}$ Department of Rehabilitation, Physical and Sports Medicine, Institute of Public Health, Vilnius University, Faculty of Medicine, Lithuania

\section{Introduction}

Multiple Sclerosis (MS) impairs levels of work capacity and causes substantial impact on personal quality of life as well as on the welfare of his/her family.

The aim of this study was is to evaluate the informative capability of the International Classification of Functioning, Disability and Health (ICF) in regard to different clinical methods used to assess level of disability and/or work capacity of patients with MS.

\section{Methods}

181 persons with MS were assessed at Neurology centre Vilnius University hospital Santaros klinikos.

Expanded Disability Status Scale (EDSS (1)) was used to evaluate level of work capacity. Self-reports were also collected according to Brief ICF Core Set for Multiple Sclerosis, Hospital Anxiety and Depression Scale (HAD-A (2), HAD-D (3)), Fatigue Descriptive Scale (FDS (4)), Short Form 36 Medical Outcomes Study questionnaire (physical health - (SF36-PH (5)), mental healt - SF36-MH (6)), while cognitive impairments were assessed using California Verbal Learning Test II ed. (CVLT-II (7)), Symbol Digit Modalities Test (SDMT (8)), Brief Visuospatial Memory Test -Revised (BVMT-R (9)).

\section{Results}

Brief ICF Core Set correlations with different scales

s110 Structure of brain
\begin{tabular}{|c|c|c|c|c|c|c|c|c|}
\hline $\mathbf{1}$ & $\mathbf{2}$ & $\mathbf{3}$ & $\mathbf{4}$ & $\mathbf{5}$ & $\mathbf{6}$ & $\mathbf{7}$ & $\mathbf{8}$ & $\mathbf{9}$ \\
\hline, $200^{*}$ & $\mathrm{x}$ & $\mathrm{x}$ &, $154^{*}$ & $\mathrm{x}$ & $\mathrm{x}$ &,$- 293^{*}$ &,$- 288^{*}$ &,$- 281^{*}$ \\
\hline
\end{tabular}

S120 Spinal cord and related structures

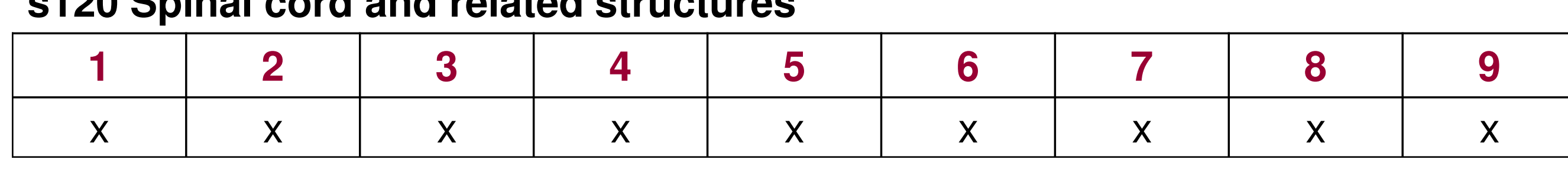

b130 Energy and drive functions

\begin{tabular}{|c|c|c|c|c|c|c|c|c|}
\hline $\mathbf{1}$ & $\mathbf{2}$ & $\mathbf{3}$ & $\mathbf{4}$ & $\mathbf{5}$ & $\mathbf{6}$ & $\mathbf{7}$ & $\mathbf{8}$ & $\mathbf{9}$ \\
\hline, $289^{*}$ &, $565^{* \prime}$ &, $404^{*}$ &, $523^{*}$ &,$- 537^{\prime \prime}$ &,$- 558^{\prime \prime}$ &,$- 223^{*}$ &,$- 242^{* *}$ &,$- 242^{*}$ \\
\hline
\end{tabular}

b152 Emotional functions

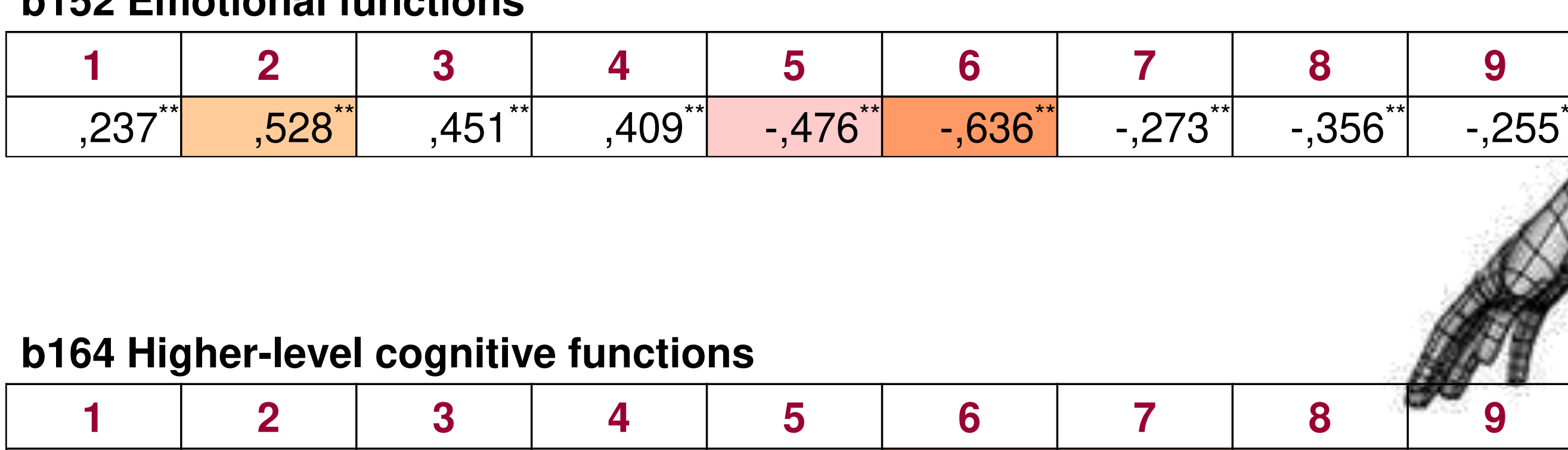

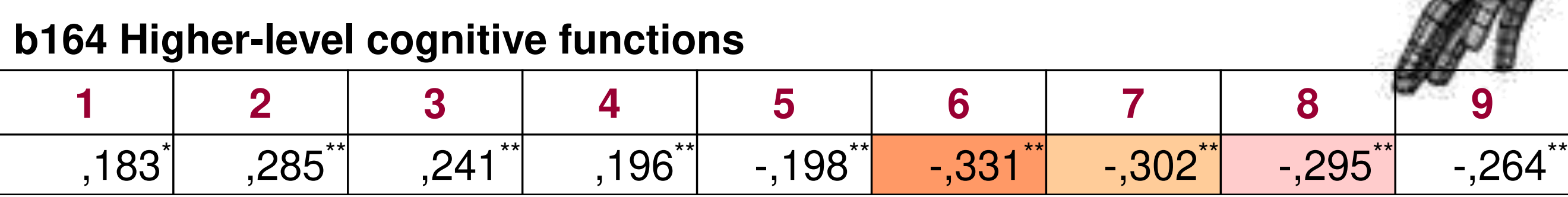

b210 Seeing functions

\begin{tabular}{|c|c|c|c|c|c|c|c|c|}
\hline 1 & 2 & 3 & 4 & 5 & 6 & 7 & 8 & 9 \\
\hline, $240^{* *}$ &, $197^{*}$ & $\mathrm{X}$ &, $262^{*}$ &,$- 166^{*}$ &,$- 180^{\circ}$ &,$- 155^{*}$ &,$- 184^{*}$ &,$- 183^{*}$ \\
\hline
\end{tabular}

b280 Sensation of pain

\begin{tabular}{|c|c|c|c|c|c|c|c|c|}
\hline $\mathbf{1}$ & $\mathbf{2}$ & $\mathbf{3}$ & $\mathbf{4}$ & $\mathbf{5}$ & $\mathbf{6}$ & $\mathbf{7}$ & $\mathbf{8}$ & $\mathbf{9}$ \\
\hline, $231^{*}$ &, $251^{*}$ &, $193^{* *}$ &, $316^{* *}$ &,$- 497^{* *}$ &,$- 276^{* *}$ & $\mathrm{x}$ &,$- 149^{*}$ & $\mathrm{x}$ \\
\hline
\end{tabular}

b620 Urination functions

\begin{tabular}{|c|c|c|c|c|c|c|c|c|}
\hline $\mathbf{1}$ & $\mathbf{2}$ & $\mathbf{3}$ & $\mathbf{4}$ & $\mathbf{5}$ & $\mathbf{6}$ & $\mathbf{7}$ & $\mathbf{8}$ & $\mathbf{9}$ \\
\hline, $556^{*}$ &, $267^{*}$ &, $173^{*}$ &, $445^{*}$ &,$- 449^{* *}$ &,$- 310^{*}$ &,$- 205^{* *}$ &,$- 229^{*}$ &,$- 233^{* *}$ \\
\hline
\end{tabular}

\section{b730 Muscle power functions}

\begin{tabular}{|c|c|c|c|c|c|c|c|c|}
\hline $\mathbf{1}$ & $\mathbf{2}$ & $\mathbf{3}$ & $\mathbf{4}$ & $\mathbf{5}$ & $\mathbf{6}$ & $\mathbf{7}$ & $\mathbf{8}$ & $\mathbf{9}$ \\
\hline, $659^{*}$ &, $189^{*}$ & $\mathrm{X}$ &, $428^{* *}$ &,$- 582^{*}$ &,$- 345^{* *}$ &,$- 246^{*}$ &,$- 274^{* *}$ &,$- 279^{*}$ \\
\hline
\end{tabular}

b770 Gait pattern functions
\begin{tabular}{|c|c|c|c|c|c|c|c|c|}
\hline $\mathbf{1}$ & $\mathbf{2}$ & $\mathbf{3}$ & $\mathbf{4}$ & $\mathbf{5}$ & $\mathbf{6}$ & $\mathbf{7}$ & $\mathbf{8}$ & $\mathbf{9}$ \\
\hline, $676^{*}$ &, $266^{*}$ &, $176^{*}$ &, $460^{* *}$ &,$- 614^{*}$ &,$- 387^{* *}$ &,$- 229^{*}$ &,$- 319^{*}$ &,$- 215^{*}$ \\
\hline
\end{tabular}

\section{Conclusions}

Results of the study results highlight the reliability of the Brief ICF Core Set in defining working capacity level of the patients with MS. Therefore we recommend using ICF Core Set in the institutions of Lithuania to assess patients with MS.

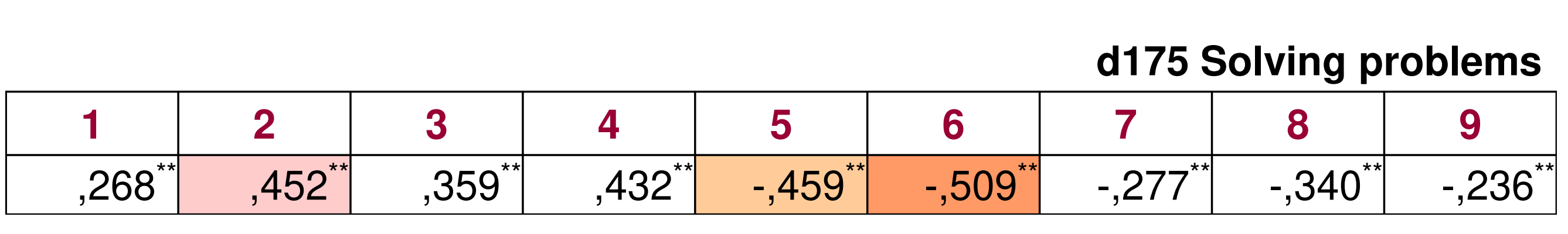

d230 Carrying out daily routine

\begin{tabular}{|l|l|l|l|l|c|c|c|c|}
\hline \multicolumn{1}{|c|}{ d230 Carrying out daily routine } \\
\hline $\mathbf{1}$ & $\mathbf{2}$ & $\mathbf{3}$ & $\mathbf{4}$ & $\mathbf{5}$ & $\mathbf{6}$ & $\mathbf{7}$ & $\mathbf{8}$ & $\mathbf{9}$ \\
\hline, $255^{*}$ &, $306^{* \prime}$ &, $163^{*}$ &, $366^{*}$ &,$- 409^{*}$ &,$- 351^{*}$ & $\mathrm{x}$ & $\mathrm{x}$ & $\mathrm{x}$ \\
\hline
\end{tabular}

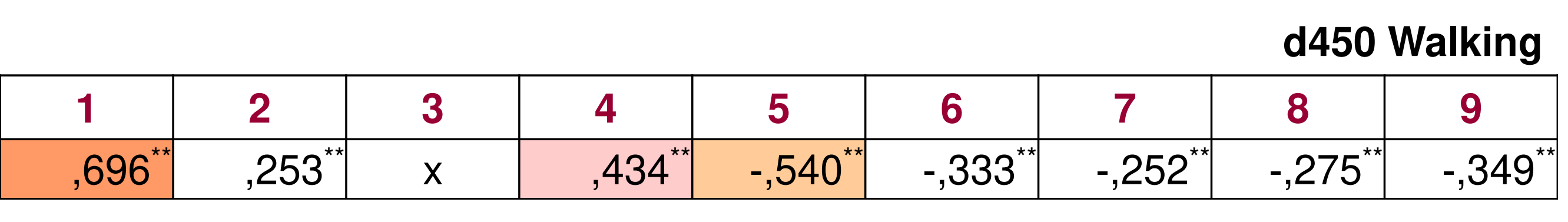

d455 Moving around \begin{tabular}{|c|c|c|c|c|c|c|c|c|}
\hline $\mathbf{1}$ & $\mathbf{2}$ & $\mathbf{3}$ & $\mathbf{4}$ & $\mathbf{5}$ & $\mathbf{6}$ & $\mathbf{7}$ & $\mathbf{8}$ & $\mathbf{9}$ \\
\hline, $556^{*}$ &, $386^{*}$ &, $215^{*}$ &, $417^{*}$ &,$- 592^{* *}$ &,$- 421^{*}$ &,$- 266^{*}$ &,$- 286^{*}$ &,$- 307^{*}$ \\
\hline
\end{tabular}

d760 Family relationships

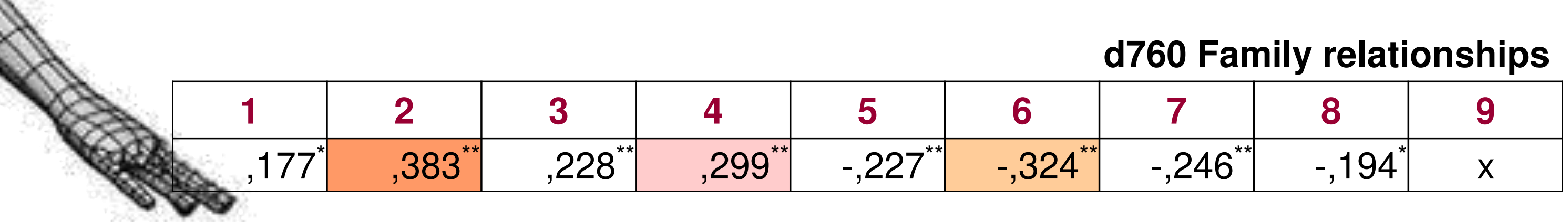

d850 Remunerative employment \begin{tabular}{|c|c|c|c|c|c|c|c|c|}
\hline $\mathbf{1}$ & $\mathbf{2}$ & $\mathbf{3}$ & $\mathbf{4}$ & $\mathbf{5}$ & $\mathbf{6}$ & $\mathbf{7}$ & $\mathbf{8}$ & $\mathbf{9}$ \\
\hline, $354^{* *}$ &, $320^{*}$ &, $272^{*}$ &, $297^{*}$ &,$- 419^{*}$ &,$- 405^{* *}$ &,$- 360^{*}$ &,$- 456^{*}$ &,$- 358^{*}$ \\
\hline
\end{tabular}

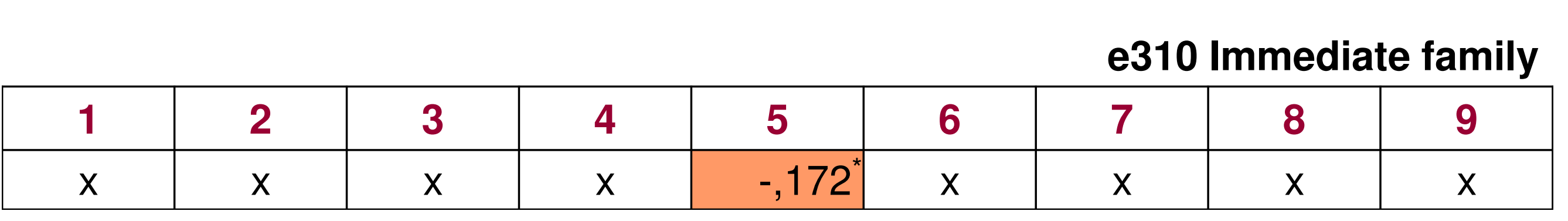

e355 Health professionals

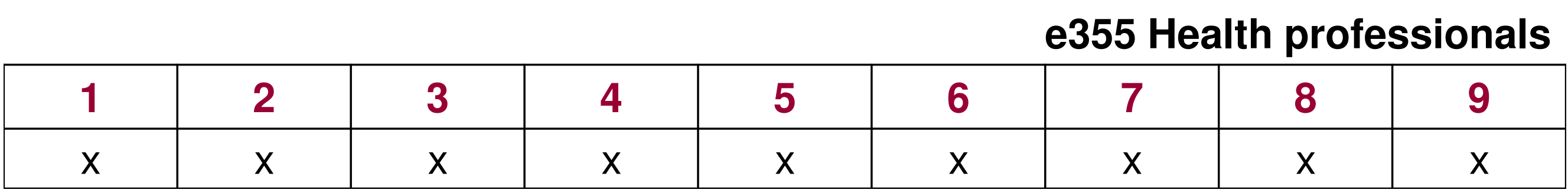

e410 Individual attitudes of immediate family members

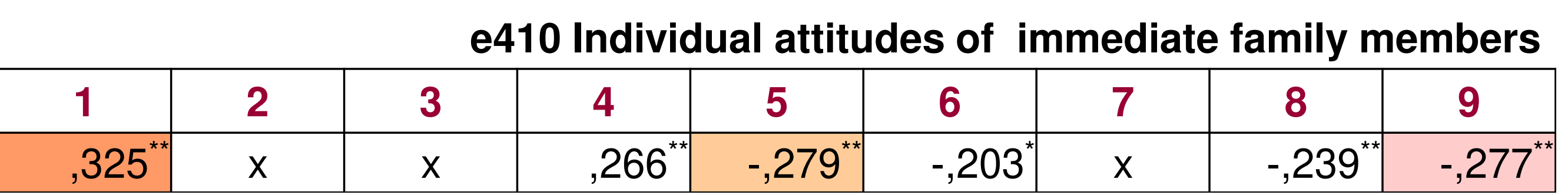

**. Correlation is significant at the 0.01 level (2-tailed)

Correlation is significant at the 0.05 level (2-tailed)

$\mathrm{X}$ - no significant correlation.

Different colours mean highest results in concrete ICF item.

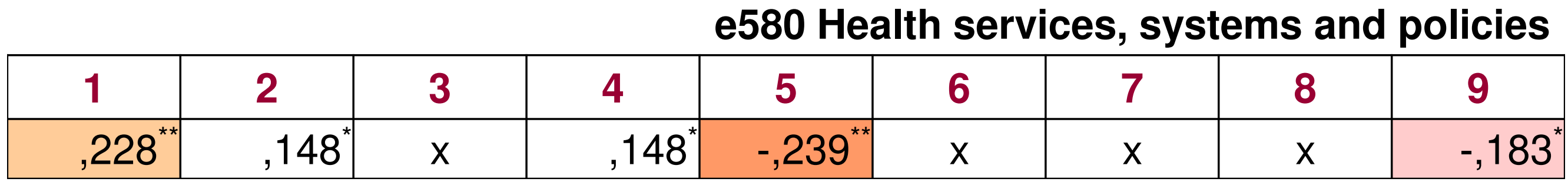

\section{References}

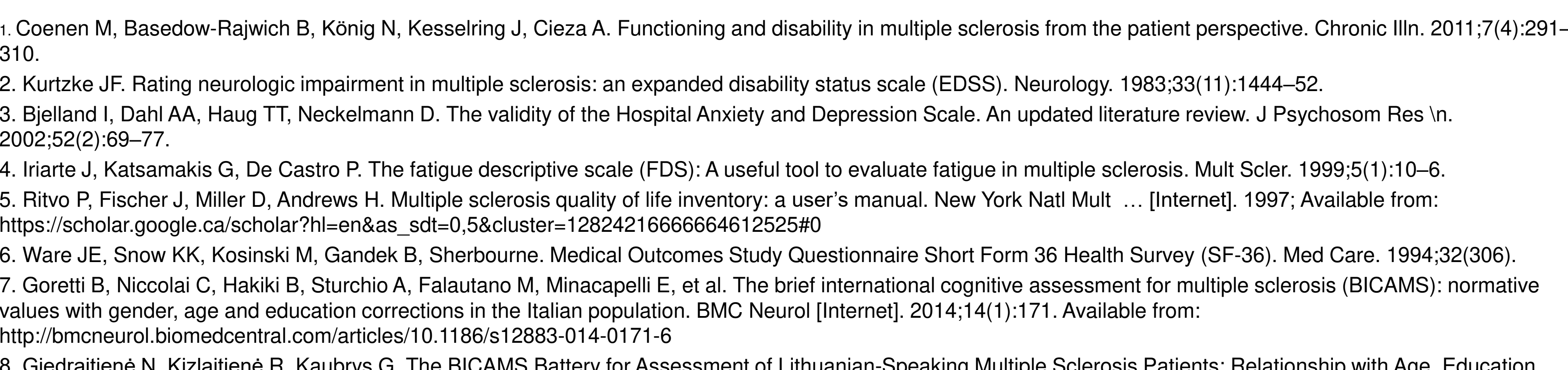

